\title{
Distribución de Liolaemus torresi (Squamata: Liolaemidae), lagarto endémico del desierto de Chile
}

\author{
Distribution of Liolaemus torresi (Squamata: Liolaemidae), endemic lizard of the Chilean desert
}

\author{
Edvin Riveros-RIFFO ${ }^{1 *}$ \& Juan C. Torres-Mura ${ }^{2}$ \\ ${ }^{1 *}$ Red de Observadores de Aves y Vida Silvestre de Chile, Julio Prado 1144 Dpto. 31, Providencia, Chile. \\ ${ }^{2}$ Unión de Ornitólogos de Chile, Mosqueto 459 Of. 103, Santiago, Chile. \\ *edvriveros@gmail.com
}

Liolaemus torresi (Núñez, Navarro, Garín, PincheiraDonoso \& Meriggio 2003), el Dragón de Torres-Mura, es un lagarto que posee una distribución restringida a la Región de Antofagasta, en el norte de Chile (Núñez et al. 2003). Hasta ahora, su rango geográfico conocido se limita a la localidad tipo, el Salar del Indio (N: 7.524.491; E: 513.054) y zonas desérticas próximas a Calama (N: 7.516.946; E: 507.692), entre los 2.430-2.485 msnm (Núñez et al. 2003, Ramírez \& Pincheira-Donoso 2005, Díaz-Páez et al. 2008). De acuerdo a la clasificación geográfica de Börgel (1983), las unidades geomorfológicas representadas en este territorio corresponden a la Precordillera de Domeyko y la Precordillera del Río Loa Superior (Fig. 1a); zona en que la vegetación está conformada por el Matorral Bajo Desértico Tropical Interior de Adesmia atacamensis y Cistanthe salsoloides, que se caracteriza por ser muy abierto y extremadamente xeromórfico (Luebert \& Pliscoff 2006). A nivel de microhábitat, L. torresi vive en terrenos arenosos, pedregosos o con costra salina, todos prácticamente desprovistos de vegetación, refugiándose en oquedades y bajo rocas, a las que corre rápidamente frente a una amenaza (Núñez et al. 2003). Aunque está incluida en la Ley de Caza (SAG 2012), su estado de conservación no se ha establecido formalmente en Chile (www.mma.gob. cl/clasificacionespecies/) ni tampoco a nivel internacional (IUCN 2014).

Entre 2009-2014 realizamos numerosas exploraciones en la Región de Antofagasta, desde la costa hasta $3.000 \mathrm{~m}$ en la cordillera, desplegando un esfuerzo de observación de más de 800 horas/hombre destinadas al reconocimiento de fauna silvestre, con énfasis en los reptiles. A través de recorridos pedestres o en vehículo a baja velocidad, más una exhaustiva búsqueda de individuos bajo rocas y refugios potenciales existentes en las áreas evaluadas (concentraciones de rocas, oquedades naturales del sustrato, cúmulos de escombros), logramos detectar la ocurrencia de $L$. torresi en localidades no documentadas previamente. Todos los individuos observados se fotografiaron, así como también los ambientes en que se detectaron, registrando además sus principales características topográficas. La asignación específica de los ejemplares fue realizada utilizando tanto los caracteres diagnósticos entregados por Núñez et al. (2003) como las descripciones completas de las diferentes especies "Phrynosaurianas" posibles de encontrar en la Región (L. stolzmanni [= Phrynosaura reichei] y L. audituvelatus $[=$ P. audituvelata $]$ ).

Reportamos seis nuevas localidades para L. torresi en la Región de Antofagasta, distantes entre 130 y 172 km lineales al oeste de su localidad tipo y en altitudes que fluctuaron entre los 790-1.500 msnm (Tabla 1). Considerando las unidades geomorfológicas (Börgel 1983), los registros se ubican en el Desierto de Atacama y la Cordillera de la Costa (Fig. 1a); a la vez que el piso de vegetación representado en estos territorios corresponden al Desierto Tropical Interior con Vegetación Escasa y la Formación del Desierto Absoluto (Luebert \& Pliscoff 2006). El microhábitat de L. torresi en las nuevas localidades se compone principalmente de quebradas y llanos medanosos, carentes de vegetación y con abundantes rocas (Fig. 1b y 1c), bajo las que excava cuevas de escasa profundidad que le sirven de refugio. A pesar del elevado esfuerzo de muestreo desarrollado en terreno, obtuvimos una extraordinariamente baja representación numérica, ya que sólo detectamos seis individuos durante todo el periodo de estudio (sólo un ejemplar por localidad). Producto de lo anterior, no efectuamos recolectas y sólo tomamos fotografías de los especímenes avistados (Fig. 1d y 1e), a excepción del ejemplar MNHNCL-4853, procedente de las cercanías de la Estación La Rioja (Tabla 1). Las nuevas localidades documentadas para $L$. torresi permiten ampliar en más de $150 \mathrm{~km}$ lineales su distribución geográfica en la Región de Antofagasta, al mismo tiempo que extienden en más de $1.500 \mathrm{~m}$ el límite altitudinal descrito previamente para la especie, situándolo en el gradiente 790-2.500 msnm. En amplias zonas del Desierto Absoluto este reptil es el único vertebrado posible de encontrar, aunque en algunos sectores es simpátrico, pero no sintópico, con Phyllodactylus gerrhopygus y Microlophus theresioides. En base a la gran 
similitud de hábitat existente entre las localidades aquí reportadas y el entorno de su terra typica, creemos que es posible documentar la presencia de L. torresi en otros lugares de la Región de Antofagasta, involucrando no sólo las serranías de la Precordillera de Domeyko y las franjas latitudinales que delimitan el Desierto de Atacama y la Cordillera de la Costa, sino que incluso en áreas próximas al litoral. Aun cuando el territorio en que ocurre $L$. torresi podría ser más amplio de lo descrito en la literatura, incluyendo nuestros hallazgos, la baja representación obtenida en los muestreos hace concluir que la especie es poco abundante en los lugares en que se distribuye, lo que sumado al actual desconocimiento que persiste sobre aspectos básicos de su biología y ecología, hace urgente la pronta determinación de su estado de conservación. Para ello, resulta fundamental el adecuado conocimiento de su distribución geográfica, siendo uno de los principales criterios utilizados por el Reglamento de Clasificación de Especies Silvestres, actualmente vigente en Chile.
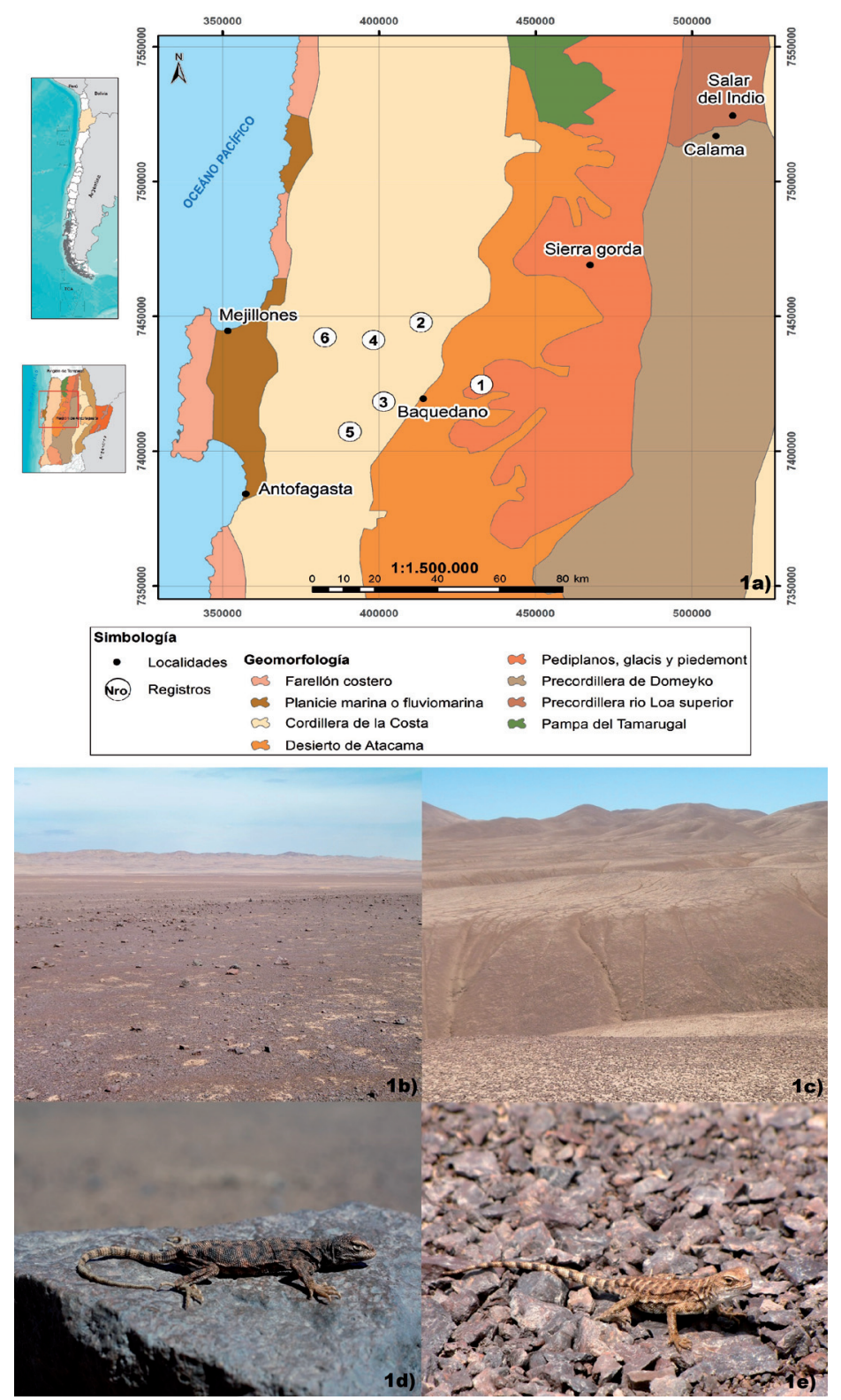

FiguRa 1. (1a). Mapa de distribución de Liolaemus torresi en la Región de Antofagasta, incluyendo las unidades geomorfológicas de Börgel (1983). Vistas panorámicas de dos nuevas localidades de L. torresi, (1b) Estación La Rioja y (1c) Rencoret. Ejemplares adultos de L. torresi procedentes de (1d) Sierra Valenzuela y (1e) Cerrillos.

Figure 1. (1a). Distribution map of Liolaemus torresi in the Antofagasta region, including geomorphological units according Börgel (1983). Panoramic views of the two new localities of L. torresi, (1b) La Rioja Station and (1c) Rencoret. Adult specimens of $L$. torresi from (1d) Sierra Valenzuela and (1e) Cerrillos. 
Tabla 1. Nuevas localidades de Liolaemus torresi en la Región de Antofagasta.

TABLE 1. New localities for Liolaemus torresi in the Antofagasta region.

\begin{tabular}{lccc}
\hline \multicolumn{1}{c}{ Localidad } & $\begin{array}{c}\text { Coordenadas } \\
\text { (UTM, WGS84) }\end{array}$ & $\begin{array}{c}\text { Altitud } \\
\text { (MSNM) }\end{array}$ & Fecha De Avistamiento \\
\hline 1 Cerrillos & $7.424 .375-428.719$ & 1.509 & Abril 2009 \\
2 Estación La Rioja & $7.447 .469-409.427$ & 1.332 & Febrero 2012 \\
3 Rencoret & $7.418 .045-397.549$ & 1.271 & Noviembre 2012 \\
4 Sierra Valenzuela & $7.440 .926-394.243$ & 1.227 & Septiembre 2009 \\
5 Mantos Blancos & $7.406 .926-386.689$ & 790 & Octubre 2010 \\
6 Quebrada Ordoñez & $7.441 .961-378.717$ & 838 & Junio 2009 \\
\hline
\end{tabular}

Recientemente, Díaz-Vega (2014) describe dos nuevas localidades en la Región de Tarapacá asignadas a L. torresi, sin embargo, la ausencia de material de referencia que pueda ser consultado, nos impide efectuar un pronunciamiento sobre la validez taxonómica de dichos registros.

\section{AGRADECIMIENTOS}

Agradecemos a Herman Núñez el acceso a la colección del MNHNCL y su colaboración en la preparación del escrito. También agradecemos a Priscila Núñez por confeccionar la cartografía de referencia y a Fernando Díaz, Marina Lemus y Cristina Ugalde por su ayuda en terreno.

\section{BIBLIOGRAFÍA}

Börgel, R. 1983. Geografía de Chile, Tomo II, Geomorfología. Geografía de Chile, Ediciones Instituto Geográfico Militar, Santiago, 182 pp.

Díaz-PÁez, H., NúÑEz, J., NúÑez, H. \& Ortiz, J.C. 2008. Estado de conservación de anfibios y reptiles. En: Herpetología de
Chile (Eds. Vidal, M.A. \& A. Labra), pp. 233-267. Science Verlag, Santiago.

DíAz-VegA, R. 2014. Range extension of Liolaemus torresi (Núñez, Navarro, Garín, Pincheira-Donoso \& Meriggio, 2003) (Squamata: Liolaemidae) in the Atacama Desert, Chile. Check List 10(5):123-1236.

Luebert, F. \& Pliscoff, P. 2006. Sinopsis bioclimática y vegetacional de Chile. Editorial Universitaria, Santiago, $316 \mathrm{pp}$.

Núñez, H., Navarro, J., Garín, C., Pincheira-Donoso, D. \& Meriggio, V. 2003. Phrynosaura manueli y Phrynosaura torresi, nuevas especies de lagartijas para el norte de Chile (Squamata: Sauria). Boletín del Museo Nacional de Historia Natural, Chile. 52:67-88.

Ramírez, G. y D. Pincheira-Donoso. 2005. Fauna del Altiplano y Desierto de Atacama. Vertebrados de la Provincia de El Loa. Phrynosaura Ediciones, Calama, 395 pp.

Servicio Agrícola y Ganadero (SAG). 2012. Legislación sobre fauna silvestre. La Ley de Caza y su Reglamento. División de Protección de los Recursos Naturales Renovables, Santiago, 96 pp.

IUCN (World CONSERvation Union). The IUCN Red List of Threatened Species. 2014. Version 2014.2. URL: http:// www.iucnredlist.org. Accesado: Julio 14, 2014.

Recibido: 16.08 .14

Aceptado: 07.06.15 